\title{
A Note on the Orthogonality Properties of the Pseudo-Chebyshev Functions
}

\author{
Diego Caratelli ${ }^{1,2, *(1)}$ and Paolo Emilio Ricci ${ }^{3}$ \\ 1 Department of Research and Development, The Antenna Company, High Tech Campus 29, \\ 5656 AE Eindhoven, The Netherlands \\ 2 Department of Electrical Engineering, Eindhoven University of Technology, PO Box 513, \\ 5600 MB Eindhoven, The Netherlands \\ 3 Department of Mathematics, International Telematic University UniNettuno, Corso Vittorio Emanuele II 39, \\ 00186 Rome, Italy; p.ricci@uninettunouniversity.net \\ * Correspondence: d.caratelli@tue.nl
}

Received: 11 June 2020; Accepted: 30 June 2020; Published: 2 August 2020

check for updates

\begin{abstract}
A novel class of pseudo-Chebyshev functions has been recently introduced, and the relevant analytical properties in terms of governing differential equation, recurrence formulae, and orthogonality have been analyzed in detail for half-integer degrees. In this paper, the previous studies are extended to the general case of rational degree. In particular, it is shown that the orthogonality properties of the pseudo-Chebyshev functions do not hold any longer.
\end{abstract}

Keywords: Grandi curves; pseudo-Chebyshev functions; recurrence relations; differential equations; orthogonality properties

\section{Introduction}

According to a beautiful historical paper by P. Butzer and F. Jongmans [1], P.L. Chebyshev, has been the "creator in St. Petersburg of the largest pre-revolutionary school of mathematics in Russia, who permitted himself to be equated only with Archimedes". Indeed, the background of Chebyshev's work on the theory of the best approximation of continuous functions was his interest in the theory of mechanisms, an Archimedean-like approach.

In order to improve the mechanism of Watt's steam engine which converts circular to straight line motion, Chebyshev was led to the investigation of new problems in approximation. Finally, he did not only solve the problem of the best approximation of a continuous function by means of polynomials, but constructed, also, more efficient mechanisms.

In order to use the alternating property of the best approximation polynomial, that is a characteristic of the solution, he introduced a polynomial version of the cosine function, which is at present known as Chebyshev polynomial of the first kind.

In recent articles, starting from the complex version of the Bernoulli spiral [2], the properties of the pseudo-Chebyshev functions of the first and second kind [3] have been analyzed. In fact, the classical Chebyshev polynomials of the first and second kind [4-7], based on the relevant trigonometric definition, can be regarded as the polynomial form of the Rhodonea (or Grandi) curves (see Figure 1). Actually, the real and imaginary part of the Bernoulli spirals identify the Rhodonea curves of fractional indices, that often appear in natural shapes [8].

It has been noticed that, similarly to the Grandi curves, the pseudo-Chebyshev functions, which are the natural extension of the Chebyshev polynomials, can be defined for rational degrees. Of course, in the more general situation, the resulting functions are no more polynomials, but irrational functions. However, in the aforementioned articles, it has been proven that, in the case of the pseudo-Chebyshev 
functions of half-integer degree, the orthogonality properties still hold true with the same interval and weights of the corresponding Chebyshev polynomials of first and second kind.

In $[9,10]$, in connection with the other kinds of Chebyshev polynomials [11], the results have been extended to the pseudo-Chebyshev functions of the third and fourth kind of half-integer degree. In particular, the recurrence relations and differential equations valid for general rational indices have been proved in $[12,13]$, but the study on the orthogonality property was limited to the case of half-integer degrees, since this property, on the interval $[-1,1]$, in general fails.

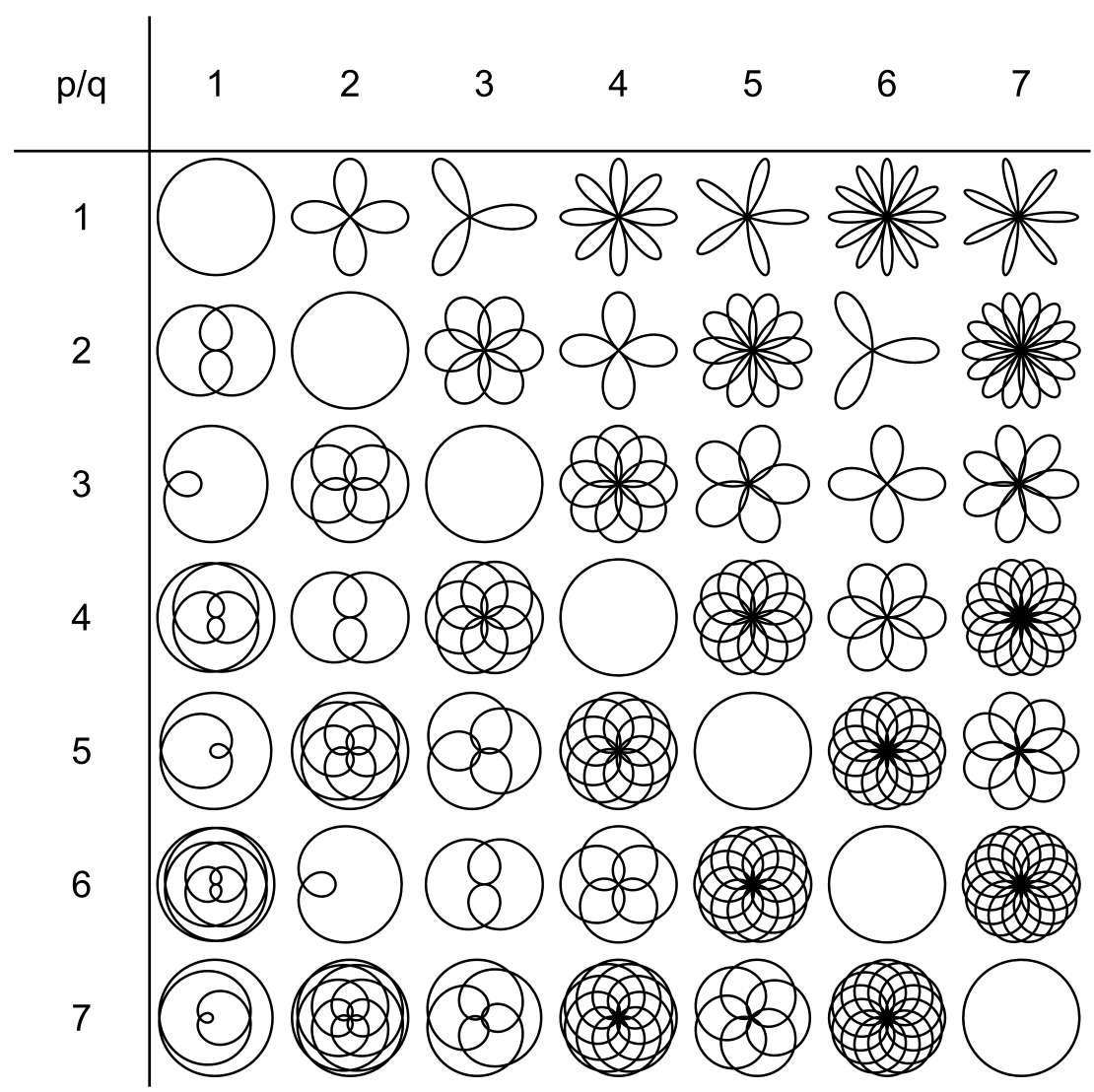

Figure 1. Rhodonea curves $\rho=\cos \left(\frac{p}{q} \theta\right)$ for different indices $k=\frac{p}{q}$.

In this article we address the following question: is it possible to define a suitable interval of the real axis, depending on a given rational degree, so that the relevant pseudo-Chebyshev functions satisfy the orthogonality condition?

We prove that this problem has no solution and, therefore, that the orthogonality property holds true for half-integer degrees only.

The article is organized as follows. In Sections 2 and 3, the definitions and main properties of the pseudo-Chebyshev functions are recalled; the relevant proofs can be found in $[2,3,9,10,12,13]$. In Section 4, we provide the answer to the question above.

\section{Definitions of Pseudo-Chebyshev Functions}

The pseudo-Chebyshev functions of the first, second, third, and fourth kind are defined, respectively, as:

$$
\begin{gathered}
T_{\frac{p}{q}}(x)=\cos \left(\frac{p}{q} \cos ^{-1} x\right), \\
\sqrt{1-x^{2}} U_{\frac{p}{q}-1}(x)=\sin \left(\frac{p}{q} \cos ^{-1} x\right),
\end{gathered}
$$




$$
\begin{gathered}
\sqrt{1-x^{2}} V_{\frac{p}{q}}(x)=\cos \left(\frac{p}{q} \cos ^{-1} x\right), \\
W_{\frac{p}{q}}(x)=\sin \left(\frac{p}{q} \cos ^{-1} x\right),
\end{gathered}
$$

with $x$ denoting a real-valued variable, and where $p$ and $q(q \neq 0)$ are integers.

Note that the definitions (1)-(4) hold even for negative indices, that is for $p / q<0$, according to the parity properties of the trigonometric functions.

In $[12,13]$, the following theorems have been proved:

Theorem 1. The pseudo-Chebyshev functions of the first kind $T_{\frac{p}{q}}(x)$ satisfy the recurrence relation:

$$
T_{\frac{p}{q}+1}(x)=2 x T_{\frac{p}{q}}(x)-T_{\frac{p}{q}-1}(x) .
$$

Theorem 2. The pseudo-Chebyshev functions of the first kind $T_{\frac{p}{q}}(x)$ satisfy the differential equation:

$$
\left(1-x^{2}\right) y^{\prime \prime}-x y^{\prime}+\left(\frac{p}{q}\right)^{2} y=0
$$

Theorem 3. The pseudo-Chebyshev functions of the second kind $U_{\frac{p}{q}}(x)$ satisfy the recurrence relation:

$$
U_{\frac{p}{q}+1}(x)=2 x U_{\frac{p}{q}}(x)-U_{\frac{p}{q}-1}(x)
$$

Theorem 4. The pseudo-Chebyshev functions of the second kind $U_{\frac{p}{q}}(x)$ satisfy the differential equation:

$$
\left(1-x^{2}\right) y^{\prime \prime}-3 x y^{\prime}+\frac{p}{q}\left(\frac{p}{q}+2\right) y=0
$$

Similar results hold for the pseudo-Chebyshev functions of the third and fourth kind, generalizing those proved in $[9,10]$ for the half-integer degree. These are recalled in the following theorems.

Theorem 5. The pseudo-Chebyshev functions of the third kind $V_{\frac{p}{q}}(x)$ satisfy the recurrence relation:

$$
V_{\frac{p}{q}+1}(x)=2 x V_{\frac{p}{q}}(x)-V_{\frac{p}{q}-1}(x) .
$$

Theorem 6. The pseudo-Chebyshev functions of the third kind $V_{\frac{p}{q}}(x)$ satisfy the differential equation:

$$
\left(1-x^{2}\right) y^{\prime \prime}-3 x y^{\prime}+\frac{p}{q}\left(\frac{p}{q}+2\right) y=0,
$$

this showing that the pseudo-Chebyshev functions of the second and third kind are independent solutions of the same homogeneous linear ordinary differential equation of the second order [14].

Theorem 7. The pseudo-Chebyshev functions of the fourth kind $W_{\frac{p}{q}}(x)$ satisfy the recurrence relation:

$$
W_{\frac{p}{q}+1}(x)=2 x W_{\frac{p}{q}}(x)-W_{\frac{p}{q}-1}(x)
$$

Theorem 8. The pseudo-Chebyshev functions of the fourth kind $W_{\frac{p}{q}}(x)$ satisfy the differential equation:

$$
\left(1-x^{2}\right) y^{\prime \prime}+x y^{\prime}+\left(\frac{p}{q}\right)^{2}\left(1-x^{2}\right) y=0
$$




\section{Orthogonality Properties of the Pseudo-Chebyshev Functions of Half-Integer Degree}

Here and in what follows, $T_{k}(x), U_{k}(x), V_{k}(x), W_{k}(x)$ denote the classical Chebyshev polynomials of the first, second, third, and fourth kind, respectively.

In the case of half-integer degrees, the definitions (1) and (2) become:

$$
\begin{gathered}
T_{k+\frac{1}{2}}(x)=\cos \left(\left(k+\frac{1}{2}\right) \cos ^{-1} x\right), \\
\sqrt{1-x^{2}} U_{k-\frac{1}{2}}(x)=\sin \left(\left(k+\frac{1}{2}\right) \cos ^{-1} x\right),
\end{gathered}
$$

whereas, for the functions of the third and fourth kind, we put:

$$
\begin{gathered}
\sqrt{1-x^{2}} V_{k+\frac{1}{2}}(x)=\cos \left(\left(k+\frac{1}{2}\right) \cos ^{-1} x\right), \\
W_{k+\frac{1}{2}}(x)=\sin \left(\left(k+\frac{1}{2}\right) \cos ^{-1} x\right) .
\end{gathered}
$$

Note that the definitions (5) and (6) hold even for negative integers, that is for $k+1 / 2<0$, thanks to the parity properties of the trigonometric functions.

The pseudo-Chebyshev functions of the first, second, third and fourth kind can be represented in terms of Chebyshev polynomials of the third and fourth kind as follows:

$$
\begin{gathered}
T_{k+\frac{1}{2}}(x)=\sqrt{\frac{1+x}{2}} V_{k}(x), \\
\sqrt{1-x^{2}} U_{k-\frac{1}{2}}(x)=\frac{1}{\sqrt{2(1+x)}} W_{k}(x), \\
\sqrt{1-x^{2}} V_{k+\frac{1}{2}}(x)=\frac{1}{\sqrt{2(1-x)}} V_{k}(x), \\
W_{k+\frac{1}{2}}(x)=\sqrt{\frac{1-x}{2}} W_{k}(x) .
\end{gathered}
$$

Orthogonality of the functions $T_{k+\frac{1}{2}}(x)$ :

$$
\int_{-1}^{1} T_{h+\frac{1}{2}}(x) T_{k+\frac{1}{2}}(x) \frac{1}{\sqrt{1-x^{2}}} d x=0,
$$

for any integers $h$ and $k$, with $h \neq k$, and:

$$
\int_{-1}^{1} T_{k+\frac{1}{2}}^{2}(x) \frac{1}{\sqrt{1-x^{2}}} d x=\frac{\pi}{2}
$$

for any integer $k$.

Orthogonality of the functions $U_{k+\frac{1}{2}}(x)$ :

$$
\int_{-1}^{1} U_{h+\frac{1}{2}}(x) U_{k+\frac{1}{2}}(x) \sqrt{1-x^{2}} d x=0 ，
$$

for any integers $h$ and $k$, with $h \neq k$, and:

$$
\int_{-1}^{1} U_{k+\frac{1}{2}}^{2}(x) \sqrt{1-x^{2}} d x=\frac{\pi}{2}
$$

for any integer $k$. 
Orthogonality of the functions $V_{k+\frac{1}{2}}(x)$ :

$$
\int_{-1}^{1} V_{h+\frac{1}{2}}(x) V_{k+\frac{1}{2}}(x) \sqrt{1-x^{2}} d x=0,
$$

for any integers $h$ and $k$, with $h \neq k$, and:

$$
\int_{-1}^{1} V_{k+\frac{1}{2}}^{2}(x) \sqrt{1-x^{2}} d x=\frac{\pi}{2},
$$

for any integer $k$.

Orthogonality of the functions $W_{k+\frac{1}{2}}(x)$ :

$$
\int_{-1}^{1} W_{h+\frac{1}{2}}(x) W_{k+\frac{1}{2}}(x) \frac{1}{\sqrt{1-x^{2}}} d x=0,
$$

for any integers $h$ and $k$, with $h \neq k$, and:

$$
\int_{-1}^{1} W_{k+\frac{1}{2}}^{2}(x) \frac{1}{\sqrt{1-x^{2}}} d x=\frac{\pi}{2},
$$

for any integer $k$.

\section{The General Orthogonality Problem}

Let us consider the following scalar product between two general pseudo-Chebyshev functions of the first kind and rational degree $p / q$ :

$$
I=\int_{a}^{b} T_{k+\frac{p}{q}}(x) T_{h+\frac{p}{q}}(x) \frac{1}{\sqrt{1-x^{2}}} d x .
$$

Upon performing the change of variable:

$$
t=\frac{\cos ^{-1} x}{q},
$$

it is straightforward to show, after simple algebraic manipulations, that:

$$
I=q \int_{\frac{\cos ^{-1}(b)}{q}}^{\frac{\cos ^{-1}(a)}{q}} \cos (m t) \cos (n t) d t,
$$

where $m=k q+p$ and $n=h q+p$. Then, we can prove the following property.

Theorem 9. A necessary and sufficient condition for the pseudo-Chebyshev functions of rational degree $p / q$ to be orthogonal in an interval of the real axis is that $q=2$.

Proof. The sufficiency of the condition is trivial. As a matter of fact, for $q=2$ we obtain the pseudo-Chebyshev functions of half-integer degree for which the result has already been proved in [9]. On the other hand, for the necessity, note that, in order for the functions $T_{k+\frac{p}{q}}(x)$ and $T_{h+\frac{p}{q}}(x)$ to be orthogonal, the identity $I=0$ has to hold true for $k \neq h$, namely $m \neq n$. Such condition is satisfied where:

$$
\frac{\cos ^{-1}(a)}{q}=\xi
$$




$$
\frac{\cos ^{-1}(b)}{q}=\xi+\pi
$$

with $\xi$ denoting an arbitrary real number. The system of Equations (7) and (8) certainly does not have real-valued solutions for $a$ and $b$ if the following inequalities are not verified simultaneously:

$$
\begin{gathered}
\left|\frac{\xi}{\pi}\right|=\left|\frac{\cos ^{-1} a}{q \pi}\right| \leq \frac{1}{|q|}, \\
\left|1+\frac{\xi}{\pi}\right|=\left|\frac{\cos ^{-1} b}{q \pi}\right| \leq \frac{1}{|q|} .
\end{gathered}
$$

By visual inspection of Figure 2, one can notice that the system of Inequalities (9) and (10) does not have solutions for $\frac{1}{|q|} \leq \frac{1}{2}$. This leads to the conclusion that a necessary condition for the orthogonality of the first kind pseudo-Chebyshev functions of rational degree $p / q$ is that $|q| \leq 2$; therefore, since $q \neq 0$ and neglecting the trivial case $q=1$, corresponding to the classical first kind Chebyshev polynomials, it has to be $q=2$.

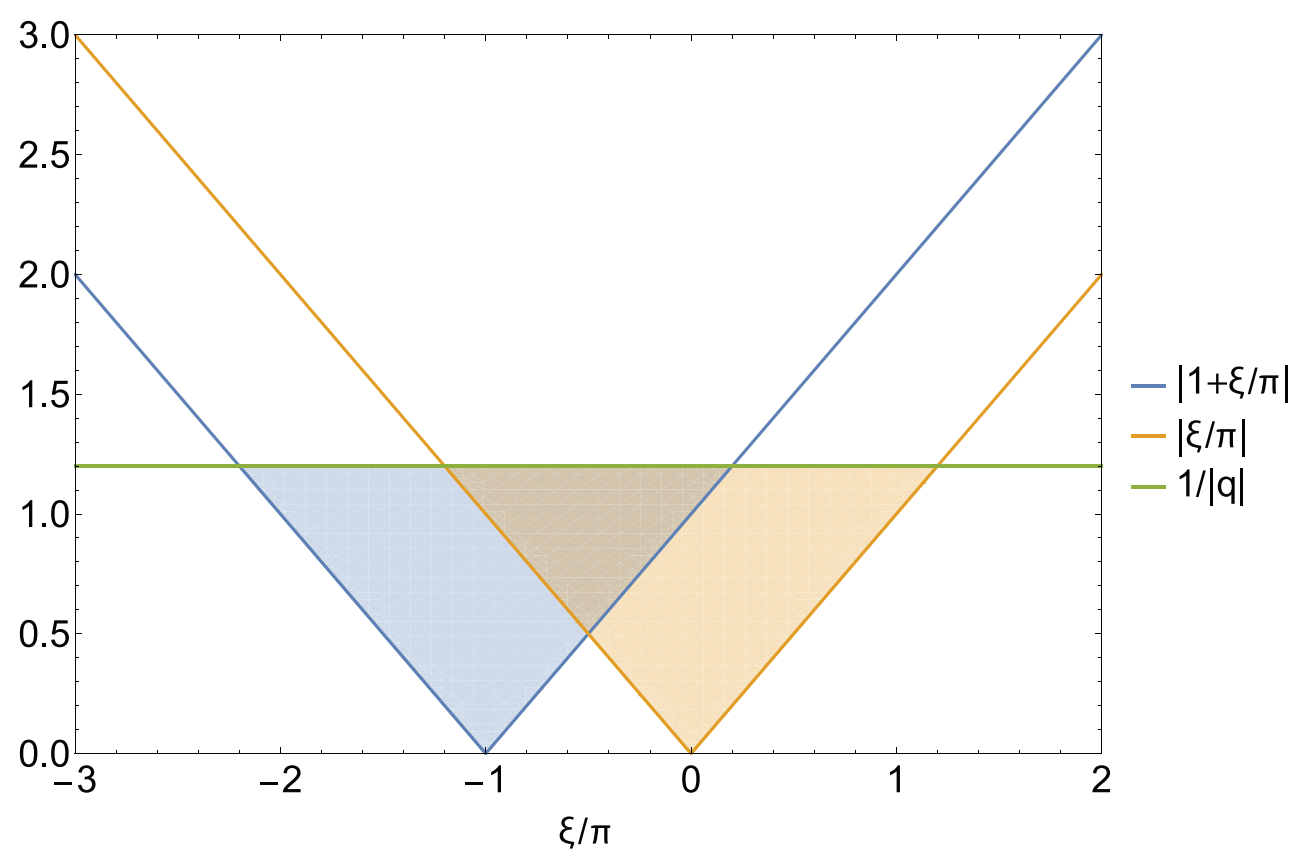

Figure 2. Graphical solution of Inequalities (9) and (10).

Remark 1. Note that the same technique can be applied in the case of pseudo-Chebyshev functions of other kinds. So, we can conclude that, even for the pseudo-Chebyshev functions of the second, third, and fourth kind, the orthogonality property holds true for the half-integer degree only.

\section{Conclusions}

We have proved that the pseudo-Chebyshev functions can satisfy the orthogonality property with respect to the classical interval and weights only for half-integer degrees. The application of the presented results to quasi-optimal function approximation and to the solution of differential problems is currently in progress and will be presented in future studies.

Author Contributions: The authors have contributed equally to this work. All authors have read and agreed to the published version of the manuscript.

Funding: This research received no external funding.

Acknowledgments: The authors are grateful to Johan Gielis for introducing them to the work of P. Butzer and F. Jongmans on the life of P.L. Chebyshev. 
Conflicts of Interest: The authors declare no conflict of interest.

\section{References}

1. Butzer, P.; Jongmans, F.P.L. Chebyshev (1821-1894): A guide to his life and work. J. Approx. Theory 1999, 96, 111-138. [CrossRef]

2. Ricci, P.E. Complex spirals and pseudo-Chebyshev polynomials of fractional degree. Symmetry 2018, $10,671$. [CrossRef]

3. Brandi, P.; Ricci, P.E. Some properties of the pseudo-Chebyshev polynomials of half-integer degree. Tbilisi Math. J. 2019, 12, 111-121. [CrossRef]

4. Mason, J.C.; Handscomb, D.C. Chebyshev Polynomials; CRC Press: New York, NY, USA, 2003.

5. Rivlin, T.J. The Chebyshev Polynomials; Wiley: New York, NY, USA, 1974.

6. Boyd, J.P. Chebyshev and Fourier Spectral Methods; Dover: New York, NY, USA, 2001.

7. Srivastava, H.M.; Manocha, H.L. A Treatise on Generating Functions; Halsted Press: New York, NY, USA, 1984.

8. Gielis, J. The Geometrical Beauty of Plants; Atlantis Press, Springer Nature: New York, NY, USA, 2017.

9. Cesarano, C.; Ricci, P.E. Orthogonality properties of the pseudo-Chebyshev functions (Variations on a Chebyshev's theme). Mathematics 2019, 7, 180. [CrossRef]

10. Cesarano, C.; Pinelas, S.; Ricci, P.E. The third and fourth kind pseudo-Chebyshev polynomials of half-integer degree. Symmetry 2019, 11, 274. [CrossRef]

11. Aghigh, K.; Masjed-Jamei, M.; Dehghan, M. A survey on third and fourth kind of Chebyshev polynomials and their applications. Appl. Math. Comput. 2008, 199, 2-12. [CrossRef]

12. Ricci, P.E. A survey on pseudo-Chebyshev functions. 4Open 2020, 3. [CrossRef]

13. Ricci, P.E. Chebyshev polynomials, rhodonea curves and pseudo-Chebyshev functions. A survey. Growth Form 2020, 1, 20-32. [CrossRef]

14. Tenenbaum, M.; Pollard, H. Ordinary Differential Equations; Dover Publications: New York, NY, USA, 1985. [CrossRef]

(C) 2020 by the authors. Licensee MDPI, Basel, Switzerland. This article is an open access article distributed under the terms and conditions of the Creative Commons Attribution (CC BY) license (http:/ / creativecommons.org/licenses/by/4.0/). 